Pacific

Journal of

Mathematics

\title{
PERIODIC SUBWORDS IN 2-PIECE WORDS
}

\section{C.M. Weinbaum}




\title{
PERIODIC SUBWORDS IN 2-PIECE WORDS
}

\author{
C.M. Weinbaum
}

We find families of words $\mathrm{W}$ where $\mathrm{W}$ is a product of $\mathrm{k}$ pieces for $\mathrm{k}=2$. For $\mathrm{k}=3,4,6, \mathrm{~W}$ arises in a small cancellation group with single defining relation $W=1$. We assume $W$ involves generators but not their inverses and does not have a periodic cyclic permutation (like XY...XYX for nonempty base word $\mathrm{XY}$ ). We prove $\mathrm{W}$ or $\mathrm{W}$ written backwards equals ABCD where ABC, CDA are periodic words with base words of different lengths. One family includes words of the form EFGG for periodic words G, E, F with the same base word and increasing lengths. Other $\mathbf{W}$ are found using Mathematica.

\section{Introduction.}

A small cancellation condition on a group's defining relations yields, for example, a solution to the conjugacy problem. See $[\mathbf{1}, \mathbf{4}]$. There are 3 types of such conditions. Each includes a condition $C(k)$ for $k=3,4$ or 6 , depending on the type. For a group $\mathrm{G}$ with one defining relation $R=1$, $C(k)$ involves the set $[R]$ of cyclic permutations of $R$ and of $R^{-1}$. A piece is a nonempty, initial subword of 2 distinct members of $[R] . C(k)$ requires that no word in $[R]$ is a product of fewer than $k$ pieces.

To study a "large cancellation" group $G$ and avoid all small cancellation types, we can use the condition that $R$ is a product of 2 pieces. What does such a word $R$ look like? For simplicity, in this paper we consider words involving generators but not their inverses. In particular, we study 2-piece words $R$, meaning

$R$ involves generators but not their inverses and $R$ is a product of 2 pieces. An attempt to classify these words led to the results in this paper. These results also lie in the field of combinatorics on words which is surveyed in $[3]$.

\section{Summary of results.}

For convenient exposition, from now on, a word $W$ is a finite sequence of letters taken from some alphabet; $|W|=$ length of $W$; the empty word=1; $|1|=0$. Write $W \sim V$ if $W, V$ are cyclic permutations. $W$ is 2-piece if 
$(\exists U, V, Y, Z \neq 1) W=U V \sim U Y \sim V Z, Z \neq U, Y \neq V .(U, V)$ is a 2piece pair. $W$ is periodic if $(\exists Q \neq 1, P, k \geq 2) W=P(Q P)^{k} . \quad X<W$ means $W=X Y, Y \neq 1$. $W$ is biperiodic if $(\exists P, Q, R, S, m, n) W=U V$, $U=P(Q P)^{m}, V=R(S R)^{n}, S R<U, Q P<V, 1 \neq Q P \neq S R \neq 1$, $U R S \neq S R U$ and $V P Q \neq Q P V ; m, n \geq 1$.

The main results are: If a 2-piece word $W$ has no periodic cyclic permutation then $W$ or $W$ written backwards is biperiodic. Each biperiodic word is 2-piece. $W$ is biperiodic and $|S|<|R S|<|P R|<P Q|<| R S R \mid$ is equivalent to $(\exists A, B, a, b, c, m, n) W=A(B A)^{b}\left(A(B A)^{c}\right)^{m}\left(A(B A)^{a}\right)^{n}$ together with $A B \neq B A, 1<a<b<c \leq 2 a, m, n \geq 1$. Such a word $W$ is not periodic for $n \geq 2$. Two other similar equivalences are proved. Other as yet unclassified biperiodic words are found using Mathematica.

The title of the paper refers to the periodic subwords $P(Q P)^{m+1}$, $R(S R)^{n+1}$ which begin word $W=P(Q P)^{m} R(S R)^{n}$ and its cyclic permutation $R(S R)^{n} P(Q P)^{m}$, respectively, whenever $\mathrm{W}$ is biperiodic and hence 2-piece.

\section{Terminology.}

Terminology in the previous section is augmented as follows. Let $A, B$ be words over some alphabet. The concatenation of words $A, B$ is written as a product $A B$. The product of $k$ copies of $A$, written $A^{k}$, is a power of $A$ if $k \geq 0$, with $A^{0}=1$, and a proper power if $k \geq 2$. Call $W$ simple if $W$ is not a proper power. Note the empty word $E=1$ is not simple since $E=E^{2}$. If $W=X Y Z$ then $X, Y, Z$ are factors of $W$; write $X, Y, Z \subseteq W . X, Z$ are left and right factors; write $X \leq W, W \geq Z$. If $U$ is a factor of $W$ then $U$ is major if $2|U| \geq|W|$ and proper if $U \neq W$. Proper left and right factors of $W$ are indicated by $X<W, W>Z$. Denote $W$ written backwards by $W^{*}$, the reverse of $W$. As in [4, p. 153], the period $\pi(W)$ of a word $W$ is the minimum length of the words admitting $W$ as a factor of some of their powers. Equivalent definitions of periodic are in Theorem 4.13. Call $W$ plain if $W$ has no periodic cyclic permutation.

We restate a definition to enable later reference to its parts:

Definition 3.1. Word $W$ is biperiodic using $U, V, P, Q, R, S, m, n$ if $W=$ $U V$ for words $U, V$ such that:

(3.1a) $\quad U=P(Q P)^{m}$ for some words $Q \neq 1, P$ and some integer $m \geq 1$.

(3.1b) $V=R(S R)^{n}$ for some words $S \neq 1, R$ and some integer $n \geq 1$.

(3.1c) $S R<U, Q P<V$.

(3.1d) $1 \neq Q P \neq S R \neq 1$.

(3.1e) $U R S \neq S R U, V P Q \neq Q P V$. 


\section{Preliminaries.}

Let $A, B, \ldots$ denote words and $a, b, \ldots$ denote integers in the following Lemmas. Lemmas 4.1-4.3 are found in Propositions 1.3.4, 8.1.1 and Theorem 8.1.2 in [3]. Lemmas 4.4, 4.5, 4.6, 4.8, 4.9, 4.10, 4.12 are in the following Propositions in [2]: 1.2, 1.3, 1.4, 1.4" $, 1.8,1.16,1.23$. Prove Lemma 4.7 from Lemma 4.6 and Lemma 4.11 from Lemma 4.10 using reverse words. Use $S$ simple if and only if $S^{*}$ simple.

Lemma 4.1. $Y, Z \neq 1, X Z=Y X$ imply $(\exists n \geq 0, U, V) Y=U V, Z=V U$, $X=U(V U)^{n}$.

Lemma 4.2. $\pi(W)=\operatorname{Min}\{|W|-|V|\}$ where $V<W>V$.

Lemma 4.3. $p=\pi(X Y), q=\pi(Y Z), d=\operatorname{gcd}(|X|,|Y|),|Y| \geq p+q-d$ imply $p=q=\pi(X Y Z)$.

Lemma 4.4. If $X Y=Y X$ then $(\exists S$ simple, $a, b \geq 0) X={ }^{a}, Y={ }^{b}$.

Lemma 4.5. If $S, T$ simple, $S^{a}=T^{b}, a, b \geq 1$ then $S=T$.

Lemma 4.6. If $S$ is a simple word and $P S \leq S^{n}, n \geq 1$ then $P$ is a power of $S$.

Lemma 4.7. If $S$ is a simple word and $S^{n} \geq S P, n \geq 1$ then $P$ is a power of $S$.

Lemma 4.8. If $P B A \leq A(B A)^{r}, r \geq 1, P \neq 1$ with $B A$ simple then $(\exists e \geq 0) P=A(B A)^{e}$.

Lemma 4.9. A cyclic permutation of a simple word is a simple word.

Lemma 4.10. $X \leq Y^{e} X, e>0, Y \neq 1$ imply $(\exists t \geq 0, E) t, E$ unique, $X=Y^{t} E, E<Y$.

Lemma 4.11. $X Y^{e} \geq X, e>0, Y \neq 1$ imply $(\exists t \geq 0, E) t, E$ unique, $X=E Y^{t}, Y>E$.

Lemma 4.12. If $X Y Z=Z Y X, X \neq 1, Z \neq 1$ then $(\exists a, b, c \geq 0, U, V)$ $X=U(V U)^{a}, Y=V(U V)^{b}, Z=U(V U)^{c}$ and the word $U V$ is simple.

Lemma 4.13. Equivalent conditions on a word $W$ are:

(4.13a) $W$ has a proper major left factor which is also a right factor.

(4.13b) $W=Y X=X Z,|X| \geq|Y|>0$.

(4.13c) $\quad(\exists k \geq 2, U \neq 1) W \leq U^{k},|W| \geq 2|U|$.

(4.13d) $W$ is periodic, that is, $(\exists B \neq 1, A, m \geq 2) W=A(B A)^{m}$.

(4.13e) $|W| \geq 2 \pi(W)$ and $W \neq 1$.

Proof. (a) if and only if (b): Use definitions. 
(b) implies (c): Deduce $|W|=|Y X| \geq|Y Y|=2|Y|$ and $W<Y W$. By Lemma 4.10, $W=Y^{t} E<Y^{t+1}$ for some word $E$ with $t \geq 1$ because $|E|<|Y|<|W|$.

(c) implies (d): From (c), $(\exists t \geq 2) t|U| \leq|W|<(1+t)|U|$. So $(\exists B \neq$ 1, $A) U=A B, W=U^{t} A$, proving (d).

(d) implies (b): Use $X=A(B A)^{m-1}, Y=A B, Z=B A$.

(d) implies (e): From (d), $W<(A B)^{m+1}$ and so $\pi(W) \leq|A B|$. It follows that $|W|=\left|A(B A)^{m}\right| \geq m|B A| \geq 2|A B| \geq 2 \pi(W)$, yielding (e).

(e) implies (c): In general, $\pi(W) \leq|W|$. From (e), $(\exists V \neq 1, k \geq 1)$ $|V|=\pi(W), W \subseteq V^{k} . \quad k \geq 2$ since $2|V|=2 \pi(W) \leq|W| \leq k|V|$. Then $(\exists U \sim V) W \leq U^{k}$.

Lemma 4.14. If $W=X Y^{e} Z, Z \leq Y \geq X, Y \neq 1, e \geq 1$ then $(\exists B \neq$ $1, A, p) 0 \leq p \leq 2, W=A(B A)^{e+p},|A B|=|Y|, X Z=A(B A)^{p}$.

Proof. $(\exists C, D) Y=Z C=D X$. Let $Y_{1}=X D, X_{1}=X Z$. Then $X_{1} \leq W=$ $\left(Y_{1}\right)^{e} X_{1}$. Apply Lemma 4.10 to $X_{1} \leq\left(Y_{1}\right)^{e} X_{1}$. $(\exists p \geq 0, A) X_{1}=\left(Y_{1}\right)^{p} A$, $A<Y_{1}$. So $(\exists B \neq 1) Y_{1}=A B$. Hence $W=(A B)^{e}(A B)^{p} A=A(B A)^{e+p}$; $X Z=X_{1}=(A B)^{p} A=A(B A)^{p}$. Also $p \leq 2$ since $\left|Y^{p} A\right|=\left|\left(Y_{1}\right)^{p} A\right|=$ $\left|X_{1}\right|=|X Z| \leq\left|Y^{2}\right|$.

Lemma 4.15. If $A B \neq B A$ then $(\exists C, D \neq 1, a, b \geq 0) C D$ simple, $A=$ $C(D C)^{a}, B=D(C D)^{b}$. For $t \geq 0, A(B A)^{t}=C(D C)^{p(t)},(A B)^{t}=$ $(C D)^{q(t)}, p(t)=(a+b+1) t+a, q(t)=(a+b+1) t$.

Proof. ( $\exists$ simple $S, e \geq 1) A B=S^{e}$. $(\exists a, b \geq 0, C, D) S=C D, A=$ $C(D C)^{a}, B=D(C D)^{b}$. $C, D \neq 1$ else $A, B$ are powers of the same word and $A B=B A$, a contradiction.

Lemma 4.16. $Y, Z \neq 1, X Z=Y X$ imply $(\exists r \geq 0, s \geq 1, C, D) C D$ simple, $Y=(C D)^{s}, Z=(D C)^{s}, X=C(D C)^{r}$.

Proof. By Lemma 4.1, $(\exists n \geq 0, U, V) Y=U V, Z=V U, X=U(V U)^{n}$. $(\exists$ simple $S) U V$ is a power of $S .(\exists i, j \geq 0, C, D) S=C D, U=C(D C)^{i}$, $V=D(C D)^{j}$. Use $r=i+n(i+j+1), s=i+j+1$.

\section{General results.}

Each 2-piece word is simple (Theorem 5.5). If $W=U V$ is 2-piece then $U, V$ appear again as factors of cyclic permutations of $W$. If $U, V$ appear at least twice in $W$ then $W$ is periodic (Theorem 5.6). If a 2-piece word $W$ is plain then $W$ or $W^{*}$ is biperiodic (Theorem 5.8). Each biperiodic word is 2-piece (Theorem 5.9). We start with some easily verifiable remarks. 
Remark 5.1. If $P Q \neq Q P$ and $m \geq 2$ then the periodic word $W=$ $P(Q P)^{m}$ is 2-piece, by definition, using $U=P(Q P)^{m-1}, V=Q P, Y=P Q$, $Z=Q P P(Q P)^{m-2}$

Remark 5.2. A word is simple, periodic, 2-piece or biperiodic if and only if its reverse has the same property.

Remark 5.3. If $(U, V)$ is a 2-piece pair then so are $(V, U)$ and $\left(V^{*}, U^{*}\right)$.

Given a 2-piece word $W, W^{*}$ inherits properties as follows:

Remark 5.4. If a 5-tuple $(W, U, V, Y, Z)$ of words satisfies $W=U V \sim$ $Y U \sim Z V, 1 \neq U \neq Z, 1 \neq V \neq Y$ then so does $\left(W^{*}, V^{*}, U^{*}, Z^{*}, Y^{*}\right)$.

Theorem 5.5. Each 2-piece word is a simple word.

Proof. Let $W$ be 2-piece word, $W=U V \sim U Y \sim V Z, 1 \neq U \neq Z$, $1 \neq V \neq Y .(\exists A, B) W=A B, U Y=B A$. Suppose $W$ is not simple. Then $(\exists$ simple $X) W=X^{m}, m \geq 2$.

If $|X| \leq|U|$ then $(\exists C) U=X C$. So $A X \leq A U Y \leq A B A B=X^{2 m}$. By Lemma 4.6, $A$ is a power of $X$, so is $W$ and hence so is $B$. Thus $A B=B A$. Then $U V=A B=B A=U Y$ implies $V=Y$, a contradiction.

If $|X|>|U|$ then $|X| \leq|V|$. By Remark 5.3, $W^{*}=V^{*} U^{*}$ is 2-piece and $W^{*}=\left(X^{*}\right)^{m}$. Get a contradiction for $W^{*}, V^{*}, U^{*}$ as previously with $W, U, V$.

Theorem 5.6. If $U, V \neq 1$ each appear at least twice in $W=U V$ then $W$ is periodic. In other words, $W=U V=I U J=K V L$ and $U, V, I, L \neq 1$ imply $W$ is periodic.

Proof. Assume $W=U V=X U T=R V Y$ and $U, V, X, Y \neq 1$. The change in letters allows a more pleasing factorization $W=R S T$ in Case 1.

Case 1: $|X|<|U| ;|Y|<|V|$. Then $(\exists F, G) U G T=X U T=W=$ $R F V=R V Y,|U|>|X|=|G|>0,|V|>|F|=|Y|>0$. So $U=$ $R F, V=G T, W=R F G T$. Let $S=F G$. Then $W=R S T$. Hence $U G T=W=R S T=W=R F V$ imply $U G=R S, S T=F G$. Apply Lemma 4.2 to $W_{1}=R S, W_{2}=S T$.

So $\pi(R S) \leq|R S|-|U|, \pi(S T) \leq|S T|-|V|$ since $R S=U G=X U$, $S T=F V=V Y$. Then $|S|=|F|+|G|=|R S|-|U|+|S T|-|V| \geq$ $\pi(R S)+\pi(S T)$. By Lemma 4.3, $\pi(R S T)=\pi(R S)=\pi(S T)$. Since $|W| \geq|S| \geq \pi(R S)+\pi(S T)=2 \pi(W), W$ is periodic by (4.13e) in Lemma 4.13.

Case 2: $|U| \leq|X| ;|V| \leq|Y|$. Then $|U| \leq|U T| \leq|V|,|V| \leq|R V| \leq|U|$. So $|U|=|V|, U=V$ and hence $W=U U$ is periodic.

Case 3: $|X|<|U| ;|V| \leq|Y|$. Then $(\exists F) U F T=X U T=W$ with $|U|>|X|=|F|>0$. So $U F=X U$. By Lemma 4.16, $(\exists C, D, r \geq$ $0, s \geq 1) C D$ simple, $U=C(D C)^{r}, F=(D C)^{s}, X=(C D)^{s} \cdot r \geq 1$ 
since $|U|>|X| \geq|C D| . D C \leq V$ since $V=F T$. Thus $|W| \geq|U F| \geq$ $2|C D|$. Also $(\exists P, Q) U=P V Q$.

Then $P D C \leq P V \leq U=C(D C)^{r}$. Also $D C \sim C D$ implies $D C$ simple by Lemma 4.9. By Lemma 4.8, $P=C(D C)^{e}, e \geq 0$ implying $V \leq(D C)^{r-e}$. So $W \leq C(D C)^{2 r-e}$; hence $\pi(W) \leq|C D|$. Then $|W| \geq$ $2|C D| \geq 2 \pi(W)$ implies $W$ is periodic by (4.13e) in Lemma 4.13.

Case 4: $|U| \leq|X| ;|Y|<|V|$. Then $V^{*}, U^{*}$ each appear at least twice in $W^{*}=V^{*} U^{*}$. Apply Case 3 to $W^{*}$; get $W^{*}$ periodic. By Remark 5.2, $W$ is periodic.

Lemma 5.7. Let $W=U V$ be a plain word. Assume cyclic $W$ has 2nd occurrences $U^{\prime \prime}, V^{\prime \prime}$ of the words $U, V$, respectively. Then (i) $U, U^{\prime \prime}$ overlap and $V, V^{\prime \prime}$ overlap and (ii) $U^{\prime \prime}$ is a factor of one of the words $U V, V U$ and $V^{\prime \prime}$ is a factor of the other.

Proof. First prove results (1)-(7).

(1) Conclusions for $U, V, U^{\prime \prime}, V^{\prime \prime}$ apply to $V^{*}, U^{*},\left(V^{\prime \prime}\right)^{*},\left(U^{\prime \prime}\right)^{*}$. By Remarks 5.2, 5.3, 5.4, assumptions on $W, U, V, U^{\prime \prime}, V^{\prime \prime}$ apply to $W^{*}, V^{*}, U^{*}$, $\left(V^{\prime \prime}\right)^{*},\left(U^{\prime \prime}\right)^{*}$, respectively.

(2) Neither $U V$ nor $V U$ has both factors $U^{\prime \prime}, V^{\prime \prime}$. Use Theorem 5.6.

(3) $U, U^{\prime \prime}$ overlap or $V, V^{\prime \prime}$ overlap. If not then $U$ has factor $V^{\prime \prime}, V$ has factor $U^{\prime \prime}$. Therefore $U=V, W=U U, W$ is periodic, contradicting $W$ is plain.

(4) $U, U^{\prime \prime}$ overlap implies ( $U^{\prime \prime} \subseteq U V$ or $U^{\prime \prime} \subseteq V U$ ). Suppose $U, U^{\prime \prime}$ overlap and $U^{\prime \prime}$ is not a factor of $U V$ or $V U$. Then $(\exists A, B, C \neq 1) W=$ $A B C V, U=A B C, U^{\prime \prime}=C V A$. So $C V<A B C V>C V, A B<C V A B>$ $A B$. Then $A B C V$ or $C V A B$ has a major left and right factor, namely, $C V$ or $A B$, respectively. Thus $A B C V$ or $C V A B$ is periodic by Lemma 4.13, contradicting $W$ is plain. Thus (4) is true.

(5) $V, V^{\prime \prime}$ overlap implies ( $V^{\prime \prime} \subseteq V U$ or $\left.V^{\prime \prime} \subseteq U V\right)$. Use (1), (4). Get $V^{*},\left(V^{\prime \prime}\right)^{*}$ overlap implies $\left(\left(V^{\prime \prime}\right)^{*} \subseteq V^{*} U^{*}\right.$ or $\left.\left(V^{\prime \prime}\right)^{*} \subseteq U^{*} V^{*}\right)$. This implies (5).

(6) $U, U^{\prime \prime}$ overlap implies $V, V^{\prime \prime}$ overlap. If not then $U, U^{\prime \prime}$ overlap but $V, V^{\prime \prime}$ do not. So $V^{\prime \prime} \subseteq U$. Also $U^{\prime \prime} \subseteq U V$ or $U^{\prime \prime} \subseteq V U$. Then $U, U^{\prime \prime}, V, V^{\prime \prime} \subseteq$ $U V$ (or $V U$ ), contradicting (2).

(7) $V, V^{\prime \prime}$ overlap implies $U, U^{\prime \prime}$ overlap. Use (1), (6). Therefore $V^{*},\left(V^{\prime \prime}\right)^{*}$ overlap implies $U^{*},\left(U^{\prime \prime}\right)^{*}$ overlap. This implies (7).

Now (i) follows from (3), (6), (7) and (ii) follows from (i), (2), (4), (5).

Theorem 5.8. Each plain, 2-piece word $W$ is biperiodic or its reverse is biperiodic. 
Proof. By Remarks 5.2 and 5.3, $W^{*}$ is plain, 2-piece. Since $W=U V$ satisfies the 2-piece condition, cyclic $W$ has 2 nd occurrences $U^{\prime \prime}, V^{\prime \prime}$ of the words $U, V$, respectively. By Lemma 5.7, there are 2 cases:

Case 1: $U^{\prime \prime} \subseteq U V, V^{\prime \prime} \subseteq V U$. Using the 2-piece property and Lemma 5.7 Part (i), it follows that $U V=W=U D B=C U^{\prime \prime} B, V U=V F G=$ $E V^{\prime \prime} G, U=F G, V=D B, Y=B C, Z=G E,|U|>|C|,|V|>|E|$ for some words $B, C, D, E, F, G \neq 1$. Since $|U|>|C|,|V|>|E|$, we can apply Lemma 4.1 to $U D=C U$ and $V F=E V$.

$(\exists Q \neq 1, P, m \geq 1) U=P(Q P)^{m}, C=P Q, D=Q P$ and $(\exists S \neq$ $1, R, n \geq 1) V=R(S R)^{n}, E=R S, F=S R$. Thus $U V=U D B=$ $U Q P B, Q P<V=R(S R)^{n}$ and $V U=V F G=V S R G, S R<U=$ $P(Q P)^{m}$, implying Conditions (3.1a), (3.1b) and (3.1c).

If $|P Q|=|R S|$ then $Q P<V=R(S R)^{n}$ implies $Q P=R S$. So $W=P X^{m+n} R$ for $X=Q P$ and $W$ is periodic by Lemma 4.14, a contradiction. So (3.1d) is true.

If $U R S=S R U$ then $F U=S R U=U R S=U E=F G E=F Z$ and $U=Z$, not true. If $Q P V=V P Q$ then $D V=Q P V=V P Q=$ $D B C=D Y$ and $V=Y$, not true. So $U R S \neq S R U, Q P V \neq V P Q$, (3.1e) is true, making $W$ biperiodic.

Case 2: $V^{\prime \prime} \subseteq U V, U^{\prime \prime} \subseteq V U$. Then $\left(V^{\prime \prime}\right)^{*} \subseteq V^{*} U^{*},\left(U^{\prime \prime}\right)^{*} \subseteq U^{*} V^{*}$. By Remark 5.4, Case 1 applies to $W^{*}$ so $W^{*}$ is biperiodic.

Theorem 5.9. Each biperiodic word is a 2-piece word.

Proof. Let $W=U V$ be biperiodic using $P, Q, R, S, U, V, m, n$. $V U$ is biperiodic using $R, S, P, Q, V, U, n, m$. By symmetry and Remark 5.3, we may assume $|S R|<|P Q|$. By Conditions (3.1a), (3.1b) and (3.1c), $S R<P Q$ and $Q P<R(S R)^{n}$. Define $F, J$ by $S R F=P Q, Q P J=R(S R)^{n}$. We now check that $W$ satisfies the definition of being 2-piece by using $Y=J P Q$, $Z=F P(Q P)^{m-1} R S$.

$$
\begin{aligned}
U Y & =P(Q P)^{m} J P Q \sim P(Q P)^{m+1} J=U Q P J=U V \\
V Z & =R(S R)^{n} F P(Q P)^{m-1} R S \sim S R F P(Q P)^{m-1} R(S R)^{n} \\
& =P Q P(Q P)^{m-1} V=U V .
\end{aligned}
$$

If $Y=V$ then $V P Q=Q P J P Q=Q P Y=Q P V$, a contradiction. If $Z=U$ then we get a contradiction from $S R U=S R Z=P Q P(Q P)^{m-1} R S=U R S$. Thus $W$ is 2-piece.

\section{Factoring some 2-piece words.}

The 2-piece words to be factored are two types of biperiodic words. They are called biperiodic-1 and biperiodic-2 words. They are 2-piece by Theorem 5.9. 
Their factorizations are called binary-1 and binary-2 words and have factors $A, B, A B \neq B A$. See Theorems 6.10 and 6.11. A 3rd type of biperiodic word, called a biperiodic-3 word, has a cyclic permutation possessing a 3rd type of factorization, a binary-3 word (Theorem 6.12). Each binary-3 word has a binary-2 cyclic permutation (Remark 6.4). Likewise, each biperiodic-3 word has a biperiodic-2 cyclic permutation, using Theorem 6.12, Remark 6.4 and Theorem 6.11.

Results in this section, together with Remark 5.3, will show that 2-piece words can be found using the above factorizations and their reverses. More precisely, we have:

Theorem 6.1. Binary-1 and binary-2 words and their reverses are 2-piece words. Each binary-3 word has a 2-piece cyclic permutation and so does its reverse.

The types of biperiodic words and factorizations are defined as follows:

Definition 6.2. A word $W$ is biperiodic-1, biperiodic-2 or biperiodic-3 if $W$ satisfies Definition 3.1 together with (6.2a), (6.2b) or (6.2c), respectively.

$$
\begin{aligned}
& |S|<|R S|<|P R|<|P Q|<|R S R| . \\
& P=1,|S R|<|Q| . \\
& |R S| \leq|P|<|P Q| .
\end{aligned}
$$

Definition 6.3. A word $W$ is binary-1, binary-2 or binary-3 if (6.3a), (6.3b) or $(6.3 \mathrm{c})$, respectively, with $A B \neq B A$. Call such $W$ binary. Terminology for later use: $W$ is binary-1 using $A, B: A B \neq B A$ and $W$ is binary-1 for $A, B, h, i, j$. Similar terminology applies to binary-2 and binary-3.

(6.3a) $W=A(B A)^{i}\left(A(B A)^{j}\right)^{m}\left(A(B A)^{h}\right)^{n}, 1<h<i<j \leq 2 h, m, n \geq 1$.

(6.3b) $W=\left(A(B A)^{i}\right)^{m}(A B)^{j}, 1 \leq i, i+1 \leq j, m, n \geq 1$.

(6.3c) $W=\left(A(B A)^{i}\right)^{m} A(B A)^{j}, 1 \leq i, i+2 \leq j, m, n \geq 1$.

Remark 6.4. Each binary-3 word $W$ has a cyclic permutation $V$ which is binary-2. In particular, if $W$ satisfies (6.3c), use $V=\left(A(B A)^{i}\right)^{m+1}(A B)^{j-i}$.

The proof that the biperiodic- 1 and binary- 1 conditions are equivalent for a word $W$ requires 3 lemmas involving closely related conditions defined as follows:

Definition 6.5. A word $W$ is biperiodic- $1^{*}$ if $W$ satisfies (3.1a)-(3.1d) with $m=n=1$. Notice the omission of (3.1e). In other words, $W$ satisfies:

$$
\begin{gathered}
(\exists P, Q, R, S) W=P Q P R S R, \quad S R<P Q, \quad Q P<R S R, \\
|S|<|R S|<|P R|<|P Q| .
\end{gathered}
$$

Definition 6.6. $W$ is binary-1* if (6.3a) with $m=n=1$. $(A B \neq B A$ not required.) 
Lemma 6.7. Word $W$ is biperiodic-1* if and only if

$(6.7 \mathrm{a})$

$(\exists F, I, J, P, Q, R, S, T, U)$ all words $\neq 1$ except possibly $S$ and $W=P Q P R S R, S R F=P Q, Q P=R S I, R=I J, P=S T, Q=R U$.

Proof. Assume (6.5a). Then $S R<P Q, Q P<R S R$ imply $P Q=S R F$, $Q P=R S I, R=I J$ for some words $F, I, J \neq 1$. $|R S|<|P R|<|P Q|$ imply $|S|<|P|,|R|<|Q|$. Using these inequalities and $S R<P Q, Q P<R S R$ we get $P=S T, Q=R U$ for some words $T, U \neq 1$. Thus (6.7a) is true. (6.5a) follows easily from (6.7a).

Lemma 6.8. If $W$ is biperiodic- $1^{*}$ then $W$ is binary-1* using $A, B: B \neq 1$.

Proof. By Lemma 6.7, we can assume $W$ satisfies (6.7a) from which we deduce:

(1) $\quad(\exists V \neq 1) F=V U$

(2) $U P=S I$

(3) $\quad|T|<|I|$

(4) $\quad R V=T R$

(5) $\quad(\exists K \neq 1) I=T K$

(6) $\quad Q=K J F$

(7) $\quad K J F S T=T K J S T K$

(8) $\quad T K=K T$

(9) $(\exists N \neq 1, r, s \geq 1) K=N^{r}$, $T=N^{s}$

(10) $S N^{r}=S K=U S$, hence $S N^{r}>S$

(11) $J F S=T J S K$

(12) $J V U S=T J S K$

(13) $J V=T J=N^{s} J$, hence $J<N^{s} J$

(14) $(\exists t \geq 0, D) S=D N^{t}$, $N>D$

(15) $(\exists u \geq 0, L) J=N^{u} L$, $L<N$

(16) $P R=D N^{b} L$, $b=r+2 s+t+u$ since $S T R U=P R U=P Q=S R F$ and so $F>U$.

since $R U P J=Q P J=R S I J$.

since $|U S T|=|U P|=|S I|$,

$|U T|=|I|$.

since (1) and

$S R V U=S R F=P Q=S T R U$.

since (3), (4) and $R=I J$.

since $P Q=S R F=S I J F$

$=S T K J F=P K J F$.

by (5), (6) and $K J F S T=Q P$ $=I J S I=T K J S T K$.

since, from (7), $K \leq T K \geq T$.

using (8) and Lemma 4.4.

since (8), (5), (2) and $P=S T$ imply $S K T=S T K=S I=U P=U S T$.

since (7) and (8).

since (11) and (1).

using (12), (10) and (9).

by applying Lemma 4.11

to $(10) S N^{r}>S$.

by applying Lemma 4.10

to $(13) J<N^{s} J$.

since $P R=S T I J$

$$
=D N^{t} N^{s} N^{r+s} N^{u} L \text {. }
$$


(17) $U P R=D N^{c} L$,

$$
c=2 r+2 s+t+u
$$

(18) $S R=D N^{a} L$,

$$
a=r+s+t+u
$$

(19) $(\exists C, M) N=L M=C D$ since $U P R=U S T I J$

$$
=S K T I J=S K T K T J .
$$

since $S R=S I J=S T K J$

$$
=D N^{t} N^{r+s} N^{u} L \text {. }
$$

Using (18) and (19) with Lemma 4.14 and (16) and (19) with Lemma 4.14, we have:

$$
\begin{gathered}
(\exists B \neq 1, A, p \geq 0) S R=A(B A)^{a+p},|A B|=|N|, \\
D L=A(B A)^{p},|A|<|N| \text { and hence } A B=D C . \\
(\exists G \neq 1, H, q \geq 0) P R=G(H G)^{b+q},|G H|=|N|, \\
D L=G(H G)^{q},|G|<|N| \text { and hence } G H=D C .
\end{gathered}
$$

So $p|N|+|A|=|D L|=q|N|+|G|$, implying $p=q,|A|=|G|$. Then $A=G$, $B=H$ since $A B=D C=G H$. So $P R=A(B A)^{b+p}$. Similarly from (17) and (19) with Lemma 4.14, $U P R=A(B A)^{c+p}$. From (16), (17) and (18), $1<a<b<c \leq 2 a$. So $1<a+p<b+p<c+p \leq 2 a+p \leq 2 a+2 p=2(a+p)$. Let $h=a+p, i=b+p, j=c+p$.

Then $W=P Q P R S R=(P R)(U P R)(S R)=A(B A)^{i} A(B A)^{j} A(B A)^{h}$ satisfies (6.3a) for $m=n=1$. So $W$ is binary-1*.

Lemma 6.9. If $W$ is binary-1* using $A, B: A \neq 1$ then $W$ is biperiodic-1*.

Proof. Assume word $W$ satisfies (6.3a) with $A \neq 1, m=n=1$. Since $i-h \leq i+h-j$, there exists an integer $r$ with $0<i-h \leq r \leq i+h-j$. Therefore $0 \leq h-i+r, j+r \leq i+h, 0<j-h \leq i-r, j-i+r \leq h$. Also

$$
(A B)^{j-i+r}<A(B A)^{h}
$$

since $A \neq 1$.

Define $P=(A B)^{r}, Q=A(B A)^{i-r}(A B)^{j-i}, R=A(B A)^{i-r}, S=$ $(A B)^{h-i+r}$. Note that $P, Q, R>1, S \geq 1, W=P Q P R S R$.

$W$ is biperiodic- $1^{*}$ with (6.5a) true because:

$S R<P Q$ since $S R=A(B A)^{h}<A(B A)^{i}(A B)^{j-i}=P Q, h<i$.

$Q P<R S R$ since $Q P=A(B A)^{i-r}(A B)^{j-i+r}$

$$
<A(B A)^{i-r} A(B A)^{h}=R S R \text { using }(*) .
$$

$|R S|<|P R|$ since $|S|=\left|(A B)^{h-i+r}\right|<\left|(A B)^{r}\right|=|P|, A B \neq 1, h<i$.

$|P R|<|P Q|$ since $|R|=\left|A(B A)^{i-r}\right|<\left|A(B A)^{i-r}(A B)^{j-i}\right|=|Q|$.

Theorem 6.10. A word is biperiodic-1 if and only if it is binary-1. 
Proof. Assume that $W$ is biperiodic-1 with $P, Q, R, S$ as in (3.1a)-(3.1e) and (6.2a). Word $W_{1}=P Q P R S R$ is biperiodic- $1^{*}$. By Lemma 6.7, $(\exists F, I, J, T$, $U)$ satisfying Condition (6.7a). By Lemma $6.8, W_{1}$ is binary- $1^{*}$ and satisfies (6.3a) for $m=n=1$ and some $B \neq 1$. As in the proof of Lemma 6.8:

$$
\begin{gathered}
W_{1}=P Q P R S R=P R U P R S R, \\
P R=A(B A)^{i}, U P R=A(B A)^{j}, S R=A(B A)^{h} .
\end{gathered}
$$

So $W=P(Q P)^{m} R(S R)^{n}=P(R U P)^{m} R(S R)^{n}=P R(U P R)^{m}(S R)^{n}$ implying (6.3a).

If $A B=B A$ then by Lemma 4.4, $A, B$ (and hence $W$ ) are powers of the same word. So $W$ is a proper power, not simple. By Theorems 5.9 and $5.5, W$ is 2-piece and simple, a contradiction. Thus $A B \neq B A$ and $W$ is a binary-1.

Now assume $W$ is binary-1. Define $P, Q, R, S$ as in proof of Lemma 6.9 so that:

$$
1<S R<P Q, Q P<R S R .
$$

$$
(P Q)^{m}=A(B A)^{i}\left(A(B A)^{j}\right)^{m-1}(A B)^{j-i} .
$$

$$
(P Q)^{m} P R=A(B A)^{i}\left(A(B A)^{j}\right)^{m-1} A(B A)^{j}=A(B A)^{i}\left(A(B A)^{j}\right)^{m} .
$$

$W=U V$ for $U=(P Q)^{m} P, V=R(S R)^{n}$. Then (6.10a) implies (3.1a)-(3.1d) are true.

If $U R S=S R U$ then $A(B A)^{h+1} \leq A(B A)^{i}<P Q<U R S$ and $A(B A)^{h} A B$ $\leq S R P<S R U$ imply $A(B A)^{h+1}=A(B A)^{h} A B$. So $B A=A B$, a contradiction. Thus $U R S \neq S R U$. If $V P Q=Q P V$ then $V P Q>Q>(A B)^{j-i}>$ $A B, Q P V>V>S R>B A$ imply $A B=B A$, a contradiction. So $V P Q \neq Q P V,(3.1 \mathrm{e})$ is true and $W$ is biperiodic-1.

Theorem 6.11. A word is biperiodic-2 if and only if it is binary-2.

Proof. Assume $W$ is biperiodic-2. Then $U=Q^{m}, 1<S R<Q<R(S R)^{n}=$ $V$, implying $R S=S R$. By Lemma 4.4, $R=X^{r}, S=X^{s}, X \neq 1, r, s \geq 1$. Since $S R<Q<R(S R)^{n},(\exists A \neq 1, B, t \geq 0) Q=A(B A)^{r+s+t}, X=A B$. Then $W=\left(A(B A)^{i}\right)^{m}(A B)^{j}$ using $i=r+s+t, j=r+n(r+s)$. Also $X^{i}<X^{i} A=Q<R(S R)^{n}=X^{j}$ implies $i<j, i+1 \leq j$. Thus (6.3b) is true. If $A B=B A$ then by Lemma $4.4 W$ is a proper power, not simple. But $W$ is 2-piece, simple by Theorems 5.9 and 5.5 , a contradiction. So $A B \neq B A$ and $W$ is binary-2.

Now assume $W$ is binary-2. Use $Q=A(B A)^{i}, P=R=1, S=A B$, $n=j, U=Q^{m}, V=R(S R)^{n}$. Then (3.1a)-(3.1d) and (6.2b) $P=1$, $|S R|<|Q|$ are true. Suppose $U R S=S R U$. Then

$$
\left(A(B A)^{i}\right)^{m} A B=U R S=S R U=A B\left(A(B A)^{i}\right)^{m} .
$$


Hence, $A B=B A$, a contradiction. Suppose $V P Q=Q P V$. Then

$$
(A B)^{n} A(B A)^{i}=V P Q=Q P V=A(B A)^{i}(A B)^{n}
$$

hence, $A B=B A$, a contradiction. Thus (3.1e) is true and $W$ is biperiodic2 .

Theorem 6.12. Each biperiodic-3 word has a binary-3 cyclic permutation. Each binary-3 word has a biperiodic-3 cyclic permutation.

Proof. Assume $W$ is binary-3. Then $W \sim W_{1}=B A\left(A(B A)^{i}\right)^{m} A(B A)^{j-1}$. Use $P=B A, Q=A(B A)^{i-1}, R=A, S=B, n=j$. Thus (3.1a)-(3.1d) and (6.2c) are true. If $U R S=S R U$ then $U R S>A B, S R U>P=B A$ imply $A B=B A$, a contradiction. If $V P Q=Q P V$ then $A(B A)^{i} A B=$ $Q P R S<Q P V$ and $A(B A)^{i+1}<A(B A)^{j}=V<V P Q$ imply $A B=B A$, a contradiction. So (3.1e) is true and $W$ has a biperiodic-3 cyclic permutation $W_{1}$.

Now assume $W$ is biperiodic-3. Then (3.1a)-(3.1c), $|R S| \leq|P|<|P Q|$ imply $S R \leq P, R S<Q P, Q P<R(S R)^{n}$. Thus $(\exists I, J \neq 1, T) Q P=R S I$, $Q P J=R(S R)^{n}, P=S R T$.

(1) $\quad Q S R T=Q P=R S I$,

$I J=R(S R)^{n-1}$

(2) $\quad I=V T$ for some $V$,

$|V|=|Q|$

(3) $\quad$ QSRTJ $=$ VTJSR

(4) $Q=V$

(5) $\quad S R T J=T J S R$

(6) $\quad Q S R T J=R S I J$

$$
=R S V T J=R S Q T J
$$

(7) $R S Q=Q S R$

(8) $\quad(\exists a, b, c \geq 0, C, D)$

$R=C(D C)^{a}, S=D(C D)^{b}$,

$Q=C(D C)^{c}, C D$ simple

(9) $D C$ is simple; $S R=(D C)^{a+b+1}$

(10) $\quad(\exists t \geq 1) T J=(D C)^{t}$

(11) $(\exists d \geq 0, F \neq 1, G) T=(F G)^{d} F$, $D C=F G$

$R(S R)^{n}=C(D C)^{a}\left((D C)^{a+b+1}\right)^{n} \quad$ from (8).

$=C(D C)^{r}$ with

$r=a+n(a+b+1) \geq n \geq 2$ using $R(S R)^{n}=Q P J=R S I J$.

from (1).

since $Q S R T J=Q P J=R S I J$

$=R(S R)^{n}=I J S R=V T J S R$.

from (3).

from (3).

using (1), (2) and (4).

from (6).

from (7) and Lemma 4.11.

by Lemma 4.9 and (8).

from (9), (5) and Lemmas 4.4 and 4.5.

from (10).

from (8) and $|R S| \leq|P|$.

$$
=|Q S R| \leq|Q P|
$$


(14) $|Q P|<\left|R(S R)^{n}\right|$

$=\left|C(D C)^{n(a+b+1)+a}\right|$

(15) $c+1<n(a+b+1)+a=r$

(16) $Q P=C(D C)^{e} F$,

$e=a+b+c+d+2$

(17)

$(\exists B \neq 1, A, p \geq 0)$

$Q P=A(B A)^{e+p}, A B=C D$.

Let $i=e+p$ so that $i \geq 2$.

(18) $R(S R)^{n} P=C(D C)^{f} F$,

$f=r+a+b+d+1$

(19) $\quad(\exists M \neq 1, L, q \geq 0)$

$R(S R)^{n} P=L(M L)^{f+q}$,

$L M=C D$. Let $j=f+q$.

(20) $|A| \equiv|C F|$ Modulo $|D C|$;

$|A| \leq|D C|$

(21)

$|L| \equiv|C F|$ Modulo $|D C|$;

$|L| \leq|D C|$

(22) $|A|=|L|, A=L, B=M$,

$L(M L)^{j}=A(B A)^{j}$

(23)

$(j-i)|D C|=(j-i)|A B|$

$=\left|A(B A)^{j}\right|-\left|A(B A)^{i}\right|$

$=\left|R(S R)^{n} P\right|-|Q P|$

$=\left|R(S R)^{n}\right|-|Q|$

$=\left|C(D C)^{r}\right|-\left|C(D C)^{c}\right|$

$=(r-c)|D C|$

(24) $j-i=r-c>1$

and hence $i+2 \leq j$

(25) $W$ has cyclic permutation

$W_{3}=(Q P)^{m} R(S R)^{n} P$

$=\left(A(B A)^{i}\right)^{m} A(B A)^{j}$

(26) $W_{3}$ satisfies $(6.3 \mathrm{c})$ from $Q P<R(S R)^{n}$ and (12).

from (13) and (14).

by (1) $Q P=Q S R T$, (8) and (11).

by (16), $Q P=X_{1}\left(Y_{1}\right)^{e} Z_{1}$,

$X_{1}=C, Y_{1}=D C=F G, Z_{1}=F$;

apply Lemma 4.14 to

$W_{1}=X_{1}\left(Y_{1}\right)^{e} Z_{1}$.

by $P=S R T$, (8) and (11).

by (17), $R(S R)^{n} P=X_{2}\left(Y_{2}\right)^{e} Z_{2}$,

$X_{2}=C, Y_{2}=D C=F G, Z_{2}=F$;

apply Lemma 4.14 to

$W_{2}=X_{2}\left(Y_{2}\right)^{f} Z_{2}$.

from (16), (17) and $|B A|=|D C|$.

from (18), (19) and $|M L|=|D C|$.

by (20) and (21).

since (17) $A B=C D$.

from (19), (22) and (17).

from (12) and (8).

from (23) and (15).

from (17), (19) and (22).

from (17), (24) and (25).

If $A B=B A$, it follows that $W_{3}, W$ are proper powers, not simple by (25) and Lemma 4.4. However, $W$ is 2-piece, simple by Theorems 5.9 and 5.5 , a contradiction. So $A B \neq B A$ and $W_{3}$ is binary-3.

\section{Some nonperiodic binary words.}

We prove that binary words, with some restrictions on their exponents, are not periodic. Details are in Theorems 7.5, 7.6 and 7.7. In the proofs, $A B$ simple, $A, B \neq 1$ can be assumed in (6.3a)-(6.3c) instead of $A B \neq B A$ because of the following lemma. 
Lemma 7.1. For $k=1,2$ or 3 , equivalent properties for a word $W$ are:

(i) binary-k using $A, B: A B \neq B A$;

(ii) binary- $k$ using $C, D: C, D \neq 1, C D$ simple.

Proof. Assume (ii) for $W$. Then $C D \neq D C$ by Lemma 4.4. Use $A=C, B=$ $D$ to get (i). Now assume (i) for $W$. By Lemma 4.14, $(\exists C, D \neq 1, a, b \geq 0)$ $C D$ simple, $A=C(D C)^{a}, B=D(C D)^{b}$. Define $p(t)=(1+a+b) t+a$, $q(t)=(1+a+b) t$ for $t \geq 0$.

Assume $k=1$. Then $p(h)<p(i)<p(j)$ since $p(t)$ is strictly increasing. Since $j \leq 2 h, p(j) \leq p(2 h)=(1+a+b) 2 h+a \leq(1+a+b) 2 h+2 a=2 p(h)$. Also $1<p(h)$ since $1<h \leq p(h)$. So $W$ is binary- 1 for $C, D, p(h), p(i), p(j)$.

Assume $k=2$. Then $p(i)+1=(1+a+b) i+a+1 \leq(1+a+b)(j-1)+a+1=$ $q(j)-b \leq q(j)$ since $i \leq j-1.1 \leq p(i)$ since $1 \leq i \leq p(i)$. So $W$ is binary-2 for $C, D, p(i), q(j)$.

Assume $k=3$. Then $0<p(i)$ since $0<i \leq p(i)$. Since $i \leq j-2$,

$$
\begin{aligned}
p(i)+2 & =(1+a+b) i+a+2 \leq(1+a+b)(j-2)+a+2 \\
& =q(j)-a-2 b \leq q(j) .
\end{aligned}
$$

So $W$ is binary-3 for $C, D, p(i), p(j)$. Thus (ii) is true for $W$ for $k=1,2,3$.

By Lemma 7.1, binary-1 and binary-3 words are products of words $X_{k}$ defined below. Results about such products appear in the next two lemmas.

Definition 7.2. For fixed words $A, B \neq 1, A B$ simple, define $X_{k}=A(B A)^{k}$, $k \geq 0$.

Lemma 7.3. Let $G=X_{a_{1}} \ldots m X_{a_{m}}, H=X_{b_{1}} \ldots m X_{b_{n}}, 1 \leq a_{i}, 1 \leq b_{j}$, $1 \leq i \leq m, 1 \leq j \leq n$ with $2 \leq m, n$. Assume $G=H$. Then $m=n, a_{i}=b_{i}$ for $1 \leq i \leq m$.

Proof. If not, pick least integer $k \geq 1$ with $a_{k} \neq b_{k}$. Assume $a_{k}<b_{k}$ so that $X_{a_{k}} B A \leq X_{b_{k}}$. Therefore $k<m, T X_{a_{k}} A B<T X_{a_{k}} X_{a_{k+1}} \leq G$, $T X_{a_{k}} B A<T X_{b_{k}} \leq H$ for $T=X_{a_{1}} \ldots m X_{a_{k-1}}$. So $A B=B A$; hence $A B$ not simple by Lemma 4.4, a contradiction.

Lemma 7.4. Let $W=X_{a_{1}} \ldots m X_{a_{m}}, 1 \leq a_{i}, 1 \leq i \leq m, m \geq 2$. Assume $(\exists F) F \leq W \geq F$.

If $\left|X_{a_{1}}\right|<|F|$ then $(\exists s, b) F=X_{a_{1}} \ldots m X_{a_{s}} X_{b}, 1 \leq s<m, 1 \leq b \leq a_{s+1}$.

If $\left|X_{a_{m}}\right|<|F|$ then $(\exists t, c) F=X_{c} X_{a_{t}} \ldots m X_{a_{m}}, 1<t \leq m, 1 \leq c \leq a_{t-1}$.

Proof. Assume $\left|X_{a_{1}}\right|<|F|$. Using $F<W, W=X_{a_{1}} \ldots m X_{a_{m}}$ induces a factorization $F=Y_{1} \ldots m Y_{r} Z$ where $Y_{k}=A$ or $Y_{k}=B$ for $1 \leq k \leq r$ and 
$Z$ equals $P$ or $Q$ for some words $P, Q$ satisfying $1 \leq P<A, 1 \leq Q<B$. Also $r \geq 3$ since $|F|>\left|X_{a_{1}}\right|, a_{1} \geq 1$. Since $A A A, B B$ do not appear in $W=X_{a_{1}} \ldots m X_{a_{m}}, Y=Y_{r-2} Y_{r-1} Y_{r}$ equals $B A A, A A B, B A B$ or $A B A$. To prove (7.4a) it suffices to prove that $Y=A B A$ and $Z=1$.

The five cases for $Y Z$ are $B A A Q, A A B P, B A B P, A B A P, A B A Q$. As shown below, only Case 4 with $P=1$ and Case 5 with $Q=1$ can occur. So indeed $Y=A B A, Z=1$.

Case $1 B A A Q: W>F, W>B A B A$ imply $B A B A>B A A Q$. By Lemma 4.7, $A Q$ is a power of $B A$ but $|A Q|<|B A|$. So $A Q=1$, contradicting $A \neq 1$.

Case $2 A A B P$ : $W>F, W>A B A$ imply $A B A>A B P$. $A B A=$ $R A B P$ for some $R \neq 1$ with $|R P|=|A|$. So $A B A B=R A B P B$, $R A B<A B A B, A B$ simple. By Lemma 4.6, $R$ is a power of $A B$ but $|R| \leq|A|<|A B|$. So $R=1$, a contradiction.

Case $3 B A B P$ : $W>F, W>A B A$ imply $A B A>A B P$ as in Case 2.

Case $4 A B A P$ : $W>F, W>B A B A$ imply $B A B A>B A P$. By Lemma 4.7, $P$ is a power of $B A$ but $|P|<|B A|$. So $P=1$.

Case $5 A B A Q: W>F, W>B A B A$ imply $B A B A>B A Q$. By Lemma 4.7, $Q$ is a power of $B A$ but $|Q|<|B A|$. So $Q=1$.

Now assume $\left|X_{a_{m}}\right|<|F|$. So $W^{*}=\left(X_{a_{m}}\right)^{*} \ldots m\left(X_{a_{1}}\right)^{*}$ and $\left(X_{a_{t}}\right)^{*}=$ $A^{*}\left(B^{*} A^{*}\right)^{t}, t \geq 0$. $A B$ simple implies $B A$ simple by Lemma 4.9. $(B A)^{*}$ is simple by Remark 5.2. $A^{*} B^{*}$ is simple since $A^{*} B^{*}=(B A)^{*}$. Note that $F^{*} \leq$ $W^{*} \geq F^{*}$. Apply (7.4a) to $W^{*}, F^{*}$. So $(\exists t, c) F^{*}=\left(X_{a_{m}}\right)^{*} \ldots m\left(X_{a_{t}}\right)\left(X_{c}\right)^{*}$, $1<t \leq m, 1 \leq c \leq a_{t-1}$. Take reverses to get (7.4b).

Theorem 7.5. Each binary-1 word $W$ with $n \geq 2$ is not periodic.

Proof. By Lemma 7.1, Definition 7.2, $W=X_{i}\left(X_{j}\right)^{m}\left(X_{h}\right)^{n}, 1<h<i<$ $j \leq 2 h$ for some $A, B \neq 1, A B$ simple. By (4.13a), it suffices to prove that each major left factor of $W$ which is also a right factor is equal to $W$. Suppose $F \leq W \geq F, 2|F| \geq|W|$. Then $|F|>\left|X_{i}\right|,|F|>\left|X_{h}\right|$ since $\left|X_{i}\right|<\left|X_{j}\right|>\left|X_{h}\right|$. By Lemma $7.4, F=X_{i} G H X_{h}$ where $G$ is a product of one or more $X_{j}$ and $H$ is a product of one or more $X_{h}$. It also follows from Lemma 7.4 that: $X_{i} G H$ and the start of $W$ have the same $X_{k}$ factors. $G H X_{h}$ and the end of $W$ have the same $X_{k}$ factors.

$|F| \leq|W|$ implies $|G H| \leq\left|\left(X_{j}\right)^{m}\left(X_{h}\right)^{n-1}\right|$. It follows that:

(7.5c) $\quad G H$ and the start of $\left(X_{j}\right)^{m}\left(X_{h}\right)^{n-1}$ have the same $X_{k}$ factors.

(7.5d) $\quad G H$ and the end of $\left(X_{j}\right)^{m}\left(X_{h}\right)^{n-1}$ have the same $X_{k}$ factors.

(7.5c) implies $G=\left(X_{j}\right)^{m}$. (7.5d) implies $H=\left(X_{h}\right)^{n-1}$. Thus $F=W$ as required. 
Theorem 7.6. Each binary- 2 word $W$ with $m(i+1) \leq j, 3 \leq j$ is not periodic.

Proof. By Lemma 7.1 and Definition 7.2, $W=\left(X_{i}\right)^{m}(A B)^{j}, 1 \leq i, i+1 \leq j$, $m \geq 1$ for some $A, B \neq 1, A B$ simple. By (4.13a) it suffices to show that $W$ has no proper major left factor which is also a right factor. Suppose $F$ is such a factor, $2|F| \geq|W|, F<W>F$. We show this implies $A B=B A$, a contradiction.

Since $m(i+1) \leq j,\left|\left(X_{i}\right)^{m}\right|=\left|A^{m}(B A)^{m i}\right|<\left|(A B)^{m(i+1)}\right| \leq\left|(A B)^{j}\right|$. Then $2|F| \geq|W|$ and $\left|\left(X_{i}\right)^{m}\right|<\left|(A B)^{j}\right|$ imply $\left|\left(X_{i}\right)^{m}\right|<|F|$. Using $F<W$, $F$ has one of the forms:

$$
\left(X_{i}\right)^{m}(A B)^{r} P,\left(X_{i}\right)^{m}(A B)^{s} A Q,\left(X_{i}\right)^{m} R
$$

where $1 \leq r<j, 0 \leq s<j, 1 \leq P<A, 1 \leq Q<B, 1<R<A$.

Case 1. $F=\left(X_{i}\right)^{m}(A B)^{r} P: W=\left(X_{i}\right)^{m}(A B)^{j}>F, r<j$ imply $(A B)^{j}$ $>(A B)^{r} P$. By Lemma 4.7, $P=1$. Then $F>B A, W>A B$ imply $A B=B A$.

Case 2. $F=\left(X_{i}\right)^{m}(A B)^{s} A Q: W>F$ implies $(A B)^{j}>(A B)^{s} A Q$ and $(A B)^{j}>A Q$. By Lemma 4.7, $A Q$ is a power of $A B$. Since $|A Q|<$ $|A B|$, we have $A Q=1, A=1$, contradicting $A B \neq B A$. So this case cannot occur.

Case 3. $F=\left(X_{i}\right)^{m} R: W>F, 3 \leq j, 1 \leq i$ imply $(A B)^{3}>A B A R$. By Lemma 4.7, $A R=(A B)^{t}$ for some $t \geq 0$. Here $t \leq 1$ since $0<$ $|R|<|A|$. If $t=0$ then $A=1$, a contradiction. If $t=1$ then $R=B$, $F>B A, W>A B$ so that $A B=B A$.

Theorem 7.7. Each binary-3 word $W$ is not periodic.

Proof. By Lemma 7.1 and Definition $7.2, W=\left(X_{i}\right)^{m} X_{j}, 1 \leq i, i+2 \leq j$, $m \geq 1$ for some $A, B \neq 1, A B$ simple. By (4.13a) it suffices to show that $W$ has no proper major left factor which is also a right factor. Suppose $F$ is such a factor, $2|F| \geq|W|, F<W>F$. We show this implies $A B=B A$, a contradiction.

Since $|W| \geq\left|X_{i} X_{j}\right|>\left|X_{i} X_{i}\right|=2\left|X_{i}\right|$ we have $2|F| \geq|W|>2\left|X_{i}\right|$. By (7.4a), $F$ has one of the forms: $\left(X_{i}\right)^{r},\left(X_{i}\right)^{s} X_{b},\left(X_{i}\right)^{m} X_{c}$ where $1 \leq r \leq m$, $1 \leq s<m, 1 \leq b<i, 1 \leq c<j$. Thus $F$ has a right factor $X_{i} X_{a}$ for some $a, 1 \leq a<j$ and hence $F>B A X_{a}$. Also $W>A B X_{a}$. Therefore $A B=B A$.

\section{Computing possibly biperiodic words.}

Let $W=U V, S R<P(Q P)^{m}=U, Q P<R(S R)^{n}=V, 0<|R S|<|P Q|<$ $|U|, m, n \geq 1$. $W$ may be biperiodic. $p=|P Q|, q=|S R|, d=|U|-p$, $e=|V|-q$ satisfy $0<d, 0<e, q<p<q+e$. Function $g$ (see below) with 
inputs $p, q, d, e$, generates such a $W$ as a list of integers $\geq 1$. Format for $g$ comes from Mathematica software, version 3.0.

$$
\begin{aligned}
& g\left[p_{-}, q_{-}, d_{-}, e_{-}\right]:=\text {Module }[\{i, k, n=p+q+d+e, w\}, \\
& \text { If }[!((0<d) \& \&(0<e) \& \&(q<p) \& \&(p<q+e)) \text {, } \\
& \text { Return["Invalid Input"]]; } \\
& w=\text { Join [ Range }[q] \text {, Range }[n-2 q] \text {, Range }[q]] \text {; } \\
& \text { For }[k=n-q, k \geq p+d+1, k--, w[[k]]=w[[k+q]]] \text {; } \\
& \operatorname{For}[k=p+d, k \geq q+1, k--, w[[k]]=w[[k+p]]] ; \\
& \operatorname{For}[k=q, k \geq 1, k--, i=w[[k+p]] ; w=w / .(k->i)] ; w] .
\end{aligned}
$$

The observed output from $g$ is (unpredictably) either biperiodic or a proper power.

In Mathematica, Range $[q]$ is the list of positive integers from 1 to $q$. $\operatorname{Join}[a, b, c]$ concatenates lists $a, b, c$. The code $k$ - - indicates integer $k$ is decreased by 1 after each stage of a loop. $w[[i]]=i$-th element of the list $w$. The code $w=w / .(k->i)$ rewrites list $w$ by replacing each instance of the current value of $k$ in list $w$ by the current value of $i$.

\section{Examples.}

We give 2 sets of examples of biperiodic words $W=P Q P R S R$ over the alphabet $\{a, b\}$. In Example (9.1), $|P R|<|R S|, P \neq 1$. In Example (9.2), $|P Q|<|P R|, P \neq 1$. Therefore (6.2a)-(6.2c) are not true. These examples include $g[24,18,3,10]$ and $g[24,18,15,14]$ for the function $g$ defined in the previous section.

Example 9.1. $W=(C C D D C D)^{2} D(a b)^{j-i}, C=a(b a)^{i}, D=a(b a)^{j}, 0<$ $i<j \leq 2 i$.

Let $P=C, Q=C D D C D, U=P Q P=C C D D C D C, R=C D(a b)^{j-i}$, $S=C C(a b)^{j-i}, V=R S R=C D D C D D(a b)^{j-i}$. W is biperiodic because:

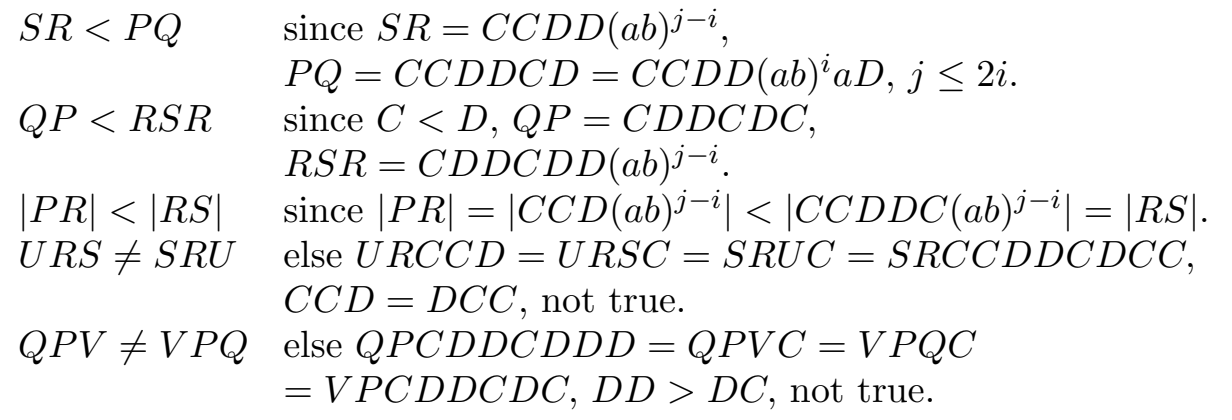

Using $i=1, j=2$ and shorthand $2=a b, 3=a b a, 5=a b a b a$, $W=(335535)(335535) 52$. Rewrite $W$ by replacing the letters $a, b$ with the symbols 1,2 , respectively. The resulting word, written as a list, is equal to $g[24,18,3,10]$. 
Example 9.2. $W=X(X Z Y)^{2} X X Y, X=(a b b)^{h} a b, Y=(a b b)^{i} a b, Z=$ $(a b b)^{2 h+1} a b, 0<h<i$.

Let $P=X X X, Q=b X(a b b)^{i-h}, P Q=X X Z(a b b)^{i-h}, Q P=b X Y X X$, $U=P Q P=X X Z Y X X, R=b X Y, S=(a b b)^{h} a, V=R S R=b X Y X X Y$. $W$ is biperiodic because:

$$
\begin{array}{ll}
S R<P Q, Q P<R S R & \text { since } S R=X X Y . \\
|P Q|<|P R| & \text { since } i-h<i \text { implies } Q<R . \\
U R S \neq S R U & \text { since } U R S>S>b a, S R U>X>a b \text { and } a b \neq b a . \\
V P Q \neq Q P V & \text { since } V P Q>Q>b b, Q P V>Y>a b \text { and } b b \neq a b .
\end{array}
$$

Using $h=1, i=2$ and shorthand 5. $=X, 8 .=Y, 11 .=Z, W=$ 5.5.11.8.5.11.8.5.5.8. Rewrite $W$ by replacing the letters $a, b$ with the symbols 1,2 , respectively. The resulting word, written as a list, is equal to $g[24,18,15,14]$.

\section{References}

[1] E. Ghys and P. de la Harpe, Sur les Groupes Hyperboliques d'apres Mikael Gromov, Birkhauser, Boston, MA, 1990 (see Appendix, in English, On small cancellation groups, by Ralph Strebel), MR 92f:53050, Zbl 731.20025.

[2] Ju.I. Hmelevskii, Equations in free semigroups, Proc. Steklov and Inst. Mat., Am. Math. Soc. Transl., 107 (1976), MR 52 \#14094, Zbl 326.02032.

[3] M. Lothaire, Combinatorics on Words, Cambridge University Press, 1997, MR 98g:68134, Zbl 874.20040.

[4] R.C. Lyndon and P.E. Schupp, Combinatorial Group Theory, Berlin and New York, Springer-Verlag, 1977, MR 58 \#28182, Zbl 368.20023.

Received May 17, 1999 and revised April 10, 2000.

Department of Mathematics

1020 Souza Drive

El Dorado Hills, CA 95762

E-mail address: swimslowly@aol.com 